\title{
Use of Gamma Encoder for Image Processing considering Human Visualization
}

\author{
Md. Zahid Hasan \\ Lecturer, Dept. of CSE \\ Green University \\ Dhaka, Bangladesh
}

\author{
T. M. Shahriar Sazzad \\ University of St Andrews
}

UK

\author{
Md. Hasibur Rahman \\ EEE, BUET \\ Dhaka, Bangladesh
}

\begin{abstract}
Image processing is a promptly developing field which finds more and more application in various information and technical systems such as: radar-tracking, communications, televisions, Biomedical image, etc. The RGB color model is standard design of computer graphics system is not ideal for all of its applications. The red, green and blue colors are highly correlated. This makes it difficult to execute the image processing algorithm. Gamma encoding of images is required to compensate for properties of human vision, to maximize the use of the bits or bandwidth relative to how humans perceive light and color. Human vision under common illumination conditions follows an approximate gamma or power function. If images are not gamma encoded, they allocate too many bits or too much bandwidth to highlights that humans cannot differentiate, and too few bits/bandwidth to shadow values that humans are sensitive to and would require more bits/bandwidth to maintain the same visual quality. Image enhancement is another technique to improve the image quality for human visualization but sometimes it does not improve the quality when the images need to be darkened or brighten. Hence, this is not a good idea to brighten images all the time when better human visualization can be obtained while darkening the images. Better human visualization is important for manual image processing which leads to compare the outcome with the semi-automated or automated one. Considering the importance of gamma encoding in image processing we propose a new method of image analysis approach which will improve visual quality for manual processing as well as will lead analyzers to analyze images automatically for comparison and testing purpose.
\end{abstract}

\section{Keywords}

HSI color model, Human Visualization, Gamma Encoder, Image processing, RGB Color model.

\section{INTRODUCTION}

Color model system is used to represent color. Moreover, it is a mathematical model which is used to describe how colors can be represented. Color space is used to describe how the components are to be interpreted. Colors can be seen as variable combinations of primary colors. Primary colors of light are additive and hence additive primary colors are red, green and blue. Combinations of $\mathrm{R}+\mathrm{G}+\mathrm{B}$ creates white. Moreover, primary colors of pigment are subtractive and hence subtractive primary colors are cyan, magenta and yellow. Combinations of $\mathrm{C}+\mathrm{M}+\mathrm{Y}$ create black [1].

There exist several methods to specify a color quantitatively, among extensively used is RGB color model where 3 different colors are added together in different ways to produce a wide range of colors. As for example for a 24 bit RGB color image, a total number of colors can be $\left(2^{8}\right)^{3}=16,777,216$.
RGB color model is used to represent and display images in electronic systems. It is to mention that RGB color model is device dependent as Red, Green and Blue levels are different from manufacturers to manufacturers [2]. Sometimes these colors vary even in same devices over a period of time and hence without a color management RGB color value does not acts as same in devices.

To improve the quality of visual perception for color images, the term image enhancement is an important factor [3]. Image enhancement is needed in many areas such as photography, scanning, image analysis etc. Image enhancement approaches fall into two broad categories such as spatial domain and frequency domain methods [4]. The term spatial domain refers to the image plane itself, and approaches in this category are based on direct manipulation of pixels in an image whereas frequency domain processing techniques are based on modifying the Fourier transform of an image.

Images can be gray-level images or color images. Comparing with color images gray-level images have got only one value for each pixel as images are made with pixel representation. There are many existing algorithm available which helps to enhance the image contrast for gray-level images considering piecewise-linear transformation function named contrast stretching with normalization, stretching with histogram techniques. Most of these available algorithm are not suitable for color images although they are used widely having poor quality and distorted effects [5].

Gray level transformation is proved to be better approach than any other transformation and hence most proposed methods are based on spatial domain approach. Image enhancement using spatial domain works with gray-level transformation or power law transformation. Power law equation is referred to as gamma.

$\mathrm{S}=\mathrm{Cr}^{\gamma}$ where $\mathrm{c}$ and $\mathrm{r}$ are positive constants. Value of $\mathrm{c}=1$ and the value of gamma can vary to set the desired result and the process used to correct power-law transformation phenomena is called gamma correction or gamma encoding.

However, it is to mention that, only enhancing the image does not improve the image quality for better visual perception. Sometimes it is needed to darken the bright images to obtain a better visualization [6]. Gamma is one of the main factor which helps to brighten or darken an image.

\section{METHODOLOGY}

We have proposed gamma encoding technique using spatial domain instead of frequency domain approach. Again, as mentioned earlier in RGB, there are three primary colors considered named Red, Green and Blue where RGB is defined as additive or subtractive model and hence different colors can be preformed using the combination of these primary colors. The RGB color model is standard design of computer 
graphics systems not ideal for all of its applications. The red, green, and blue color components are highly correlated. This makes it difficult to execute the image processing algorithms. Many processing techniques work on the intensity component of an image only. These processes are standard implemented using the HSI color model. In HSI color model, color in decomposed in hue, saturation and intensity value and thus easy for human visualization. The HSI model describes more exact color than RGB model describes for human interpretation [7].

Hue is the main attribute of a color and thus decides which color the pixel has obtained. However, hue should not be changed at any point because changing the hue changes the color as well as distortion occurs in the image. Moreover, comparing with color space like CIE LUV and CIE Lab, in HSB it is easy to control hue and color shifting. Our main approach is to preserve the hue and apply better human visualization using saturation and brightness and hence we have chosen HSI color space instead of other color space.

As mentioned earlier that image are prepared in the medical laboratory are RGB images. It is important to convert the RGB images into HSI images so that we can have hue, saturation and intensity in differently. Our main goal is to change the properties of Saturation and Intensity and preserve the hue, so we have chosen the HSI color model for better human visualization instead of choosing other color model.

\section{COLOR MODEL CONVERSION}

\subsection{RGB to HSI}

Equation (1) describes the conversion from RGB to HSI color space.

$$
\begin{aligned}
& I=\frac{1}{3}(R+G+B) \\
& S=1-\frac{3}{(R+G+B)}[\min (R, G, B)] \\
& H=\cos ^{-1}\left\{\frac{0.5\left[\left(\left(R_{-} G\right)+(R-B)\right)\right]}{\sqrt{(R-G)^{2}+(R-B)(G-B)}}\right\}
\end{aligned}
$$

If $B$ is greater than $\mathrm{G}$, then $\mathrm{H}=360^{\circ}-\mathrm{H}$

Where R, G and B are three color component of source RGB image, H, S and I it's components of hardware independent on HSI format [8].

\subsection{HSI to RGB}

As it can be seen that conversion from RGB to HSI is not easy with regard to computing algorithm complexity because it's regarding minimum from three searching (expression 1, as minimum two operators of condition), long cosine function, square root, square computation, additional operation of condition (expression 4) during one pixel conversion. More difficulty to convert from HSI color space back to standard RGB, where the process depends on which color sector $\mathrm{H}$ lies in. For the RG sector $\left(0^{0} \leq \mathrm{H} \leq 120^{\circ}\right)$, we have the following equations to convert RGB to HSI format:

$\mathrm{B}=\mathrm{I}(1-\mathrm{S})$

$$
R=I\left[1+\frac{S \cos H}{\cos \left(60^{\circ}-H\right)}\right]
$$

$\mathrm{G}=3 \mathrm{I}-(\mathrm{R}+\mathrm{B})$

For the GB sector $\left(120^{\circ} \leq \mathrm{H} \leq 240^{\circ}\right)$ :

$$
\begin{aligned}
& \mathrm{H}=\mathrm{H}-120^{\circ} \\
& \mathrm{R}=\mathrm{I}(1-\mathrm{S}) \\
& G=I\left[1+\frac{S \cos H}{\cos \left(60^{\circ}-H\right)}\right]
\end{aligned}
$$$$
B=3 I-(R+G)
$$

For the BR sector $\left(240^{\circ} \leq \mathrm{H} \leq \square 360^{\circ}\right)$ :

$$
\begin{aligned}
& \mathrm{H}=\mathrm{H}-240^{\circ} \\
& \mathrm{G}=\mathrm{I}(1-\mathrm{S}) \\
& B=I\left[1+\frac{S \cos H}{\cos \left(60^{\circ}-H\right)}\right] \\
& \mathrm{R}=3 \mathrm{I}-(\mathrm{G}+\mathrm{B})
\end{aligned}
$$

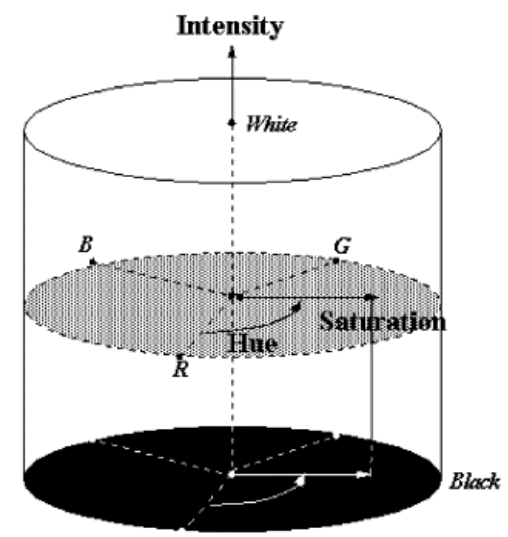

Fig 1: Cylindrical Color Space of HSI format

\section{GAMMA ENCODER}

It is wise to use luma which represents the brightness in an image and can be denoted as Y. Luma is weighted average of gamma-encoding which can be denoted as $\mathrm{Y}^{\prime}$ for $\mathrm{R}, \mathrm{G}$ and $\mathrm{B}$ and hence denoted as R'G'B'.

The equation becomes,

$\begin{array}{ll}Y=0.2126 R+0.7152 G+0.0722 B & \text { for luminance } \\ Y^{\prime}=0.2126 R^{\prime}+0.7152 G^{\prime}+0.0722 B & \text { for gamma encoding }\end{array}$

For better human visualization, the contrast enhancement operation based on the manipulation of the image histogram is histogram equalization.

Initially, we will assume a grey-scale input image, denoted $\mathrm{I}_{\text {input }}(\mathrm{X})$ If the variable $\mathrm{x}$ is continuous and normalized to lie within the range $[0,1]$, then this allows us to consider the normalized image histogram as a probability density function (PDF) $\mathrm{p}_{\mathrm{x}}(\mathrm{X})$, which defines the likelihood of given grey-scale values occurring within the vicinity of $x$. Similarly, we can denote the resulting grey-scale output image after histogram equalization as $\mathrm{I}_{\text {output }}(X)$ with corresponding $\operatorname{PDF} \operatorname{Py}(\mathrm{y})$. 
A standard result from elementary probability theory states that:

$$
p_{y}(y)=P_{x}(x)\left|\frac{d_{x}}{d_{y}}\right|
$$

which implies that the desired output PDF depends only on the known input PDF and the transformation function $\mathrm{y}=\mathrm{f}(\mathrm{x})$. Consider, then, the following transformation function, which calculates the area under the input probability density curve (i.e. integral) between 0 and an upper limit $\mathrm{x}$ :

$$
y(x)=\int_{0}^{x} p_{x}(x)^{\prime} d_{x}^{\prime}
$$

Differentiating this formula, applying Leibniz's rule and substituting into our previous statement we obtain the following:

$$
p_{y}(y)=p_{x}(x)\left|\frac{1}{p_{x}(x)}\right|
$$

Finally, because $p_{x}(x)$ is a probability density and guaranteed to be positive $\left(0 \leq \mathrm{p}_{\mathrm{x}}(\mathrm{x}) \leq 1\right)$. we can thus obtain [1]:

$$
p_{x}(y)=\frac{p_{x}(x)}{p_{y}(y)}=1 \quad 0 \leq \mathrm{y} \leq 1
$$

\section{PROCESSING STEPS}

This experiment is divided into following steps considerations for better human visualization.

1) Selection of a color image in RGB format.

2) Get the values $(r, g, b)$ for each pixel for that specific image.

3) Conversion of RGB color image to HSI color image.

4) Gamma encoding applied for brightness or darkness for better visualization.

5) Saturation value applied using histogram equalization.

6) Conversion of HSI color image to RGB color image.

7) Save and use the resultant image for other image analysis.

\section{EXPERIMENTAL RESULTS}

Histogram Equalization method applied to the original color images where this method changes the color value (hue) of the original images. It is known that majority methods of image processing working only with intensity part of color model [911]. The color model must be in full basis, it mean that model must allow to transform image to new color model, use the intensity component for image processing then return image back to RGB after processing $[12,13]$.

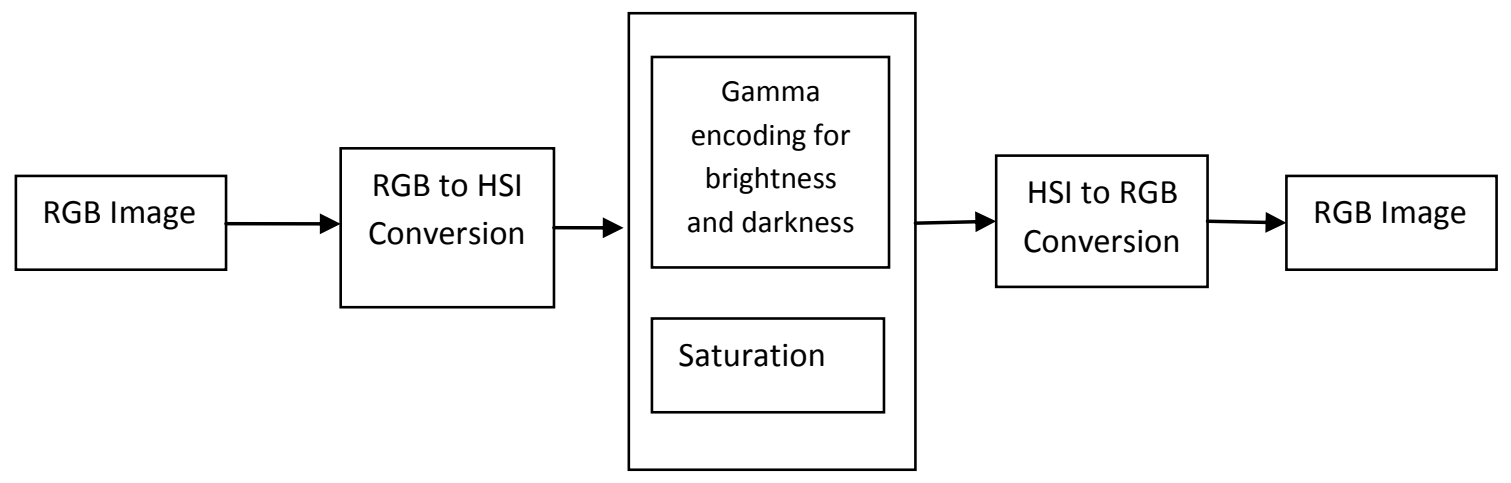

Fig 1: System Block Diagram

To evaluate the performance of our proposed method, Gamma encoding helps to maintain the visual quality of images. To evaluate the contrast performance we have applied histogram equalization saturation value from $0.4-0.6$ and gamma correction value ranges from $0.75-2.2$ in different computers as different computers acts different according to gamma value. It is to mention that gamma value $>1$ performs darkening and vice-versa.

In this section we present a hue preserving gamma encoding method based on the HSI color space. A comparison among our proposed method and the image enhancement method is carried out as shown in Fig. (1-3).
Fig. 1 shows the results for the first image, "John Coltrane" where the image enhancement technique generated a darken image (Fig. 1(b)). In turn, the image produced by our proposed method (Fig. 1(c)) is more realistic than the others. We can say that the resulted image of gamma encoding method has better quality than the others. Fig. 2(b) shows the results for the second image, the original image produces an over enhanced image, that is, the colors are very saturated. In Fig. 2(c) that our method generates an image with a good balance between non saturated and realistic colors.

From the discussion above, we claim that our method produces images (Fig (1(c)-3(c))) with the best tradeoff 


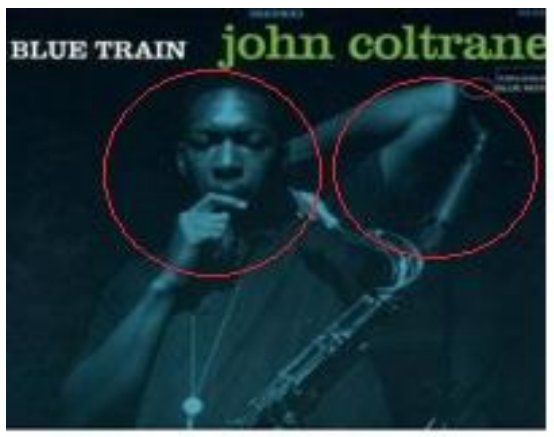

Fig 1(a): Original Image

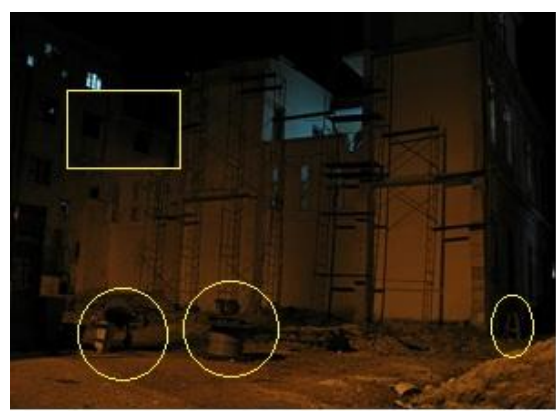

Fig 2(a): Original Image

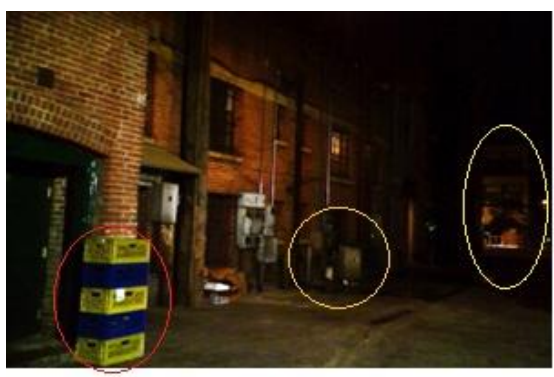

Fig 3(a): Original Image

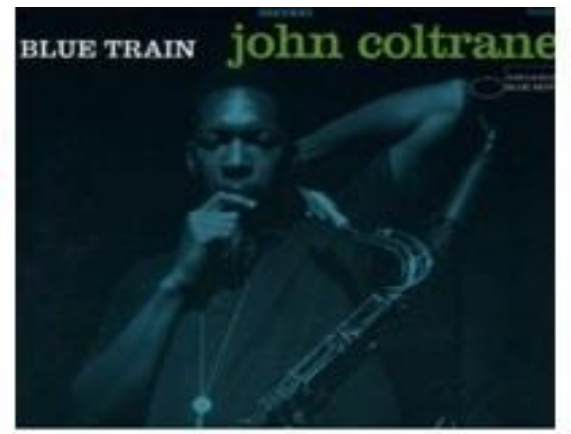

Fig 1(b): Histogram Equalization

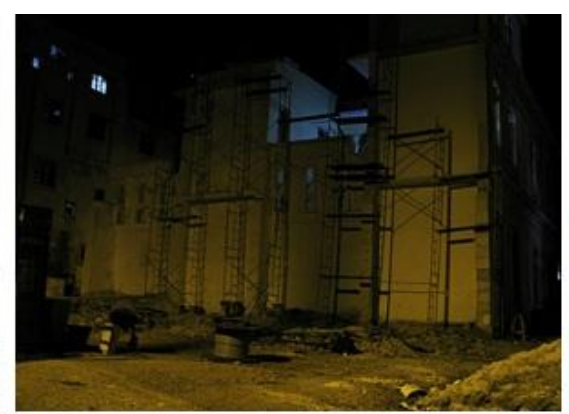

Fig 2(b): Histogram Equalization

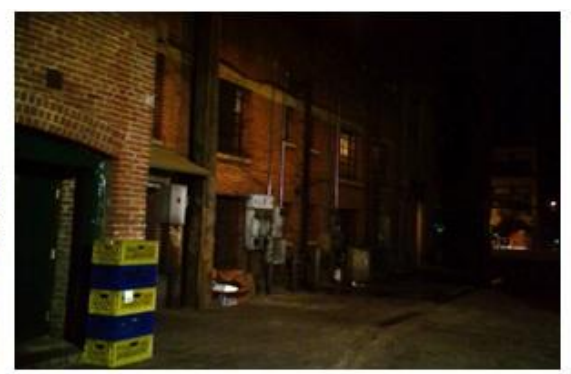

Fig 3(b): Histogram Equalization

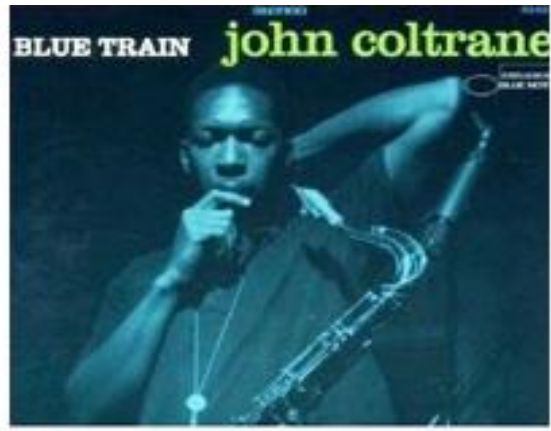

Fig 1(c): Proposed Method

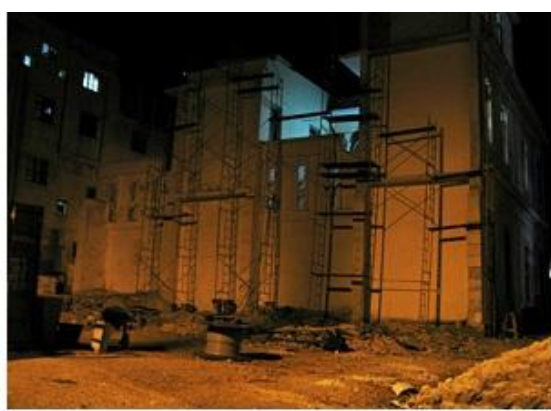

Fig 2(c): Proposed Method

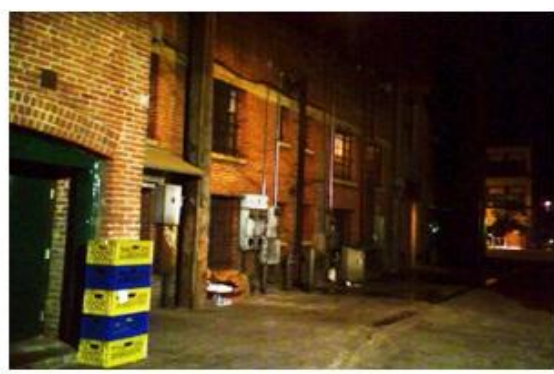

Fig 3(c): Proposed Method

Table 1: Comparison of existing and proposed method with accuracy

\begin{tabular}{|l|l|l|l|}
\hline Method Used & Number of Images & Error (\%) & Accuracy (\%) \\
\hline Existing Method & 20 & $26 \%$ & $74 \%$ \\
\hline Proposed Method & 20 & $13 \%$ & $87 \%$
\end{tabular}

between the enhanced colors and saturation. That is, our method produces images with colors that are more realistic than the image enhancement technique (which are not hue preserving), and the images are not as saturated as the ones produced by the method.

\section{CONCLUSION}

This paper has proposed a color enhancement approach using luminance component on gamma correction based on human visualization as well as saturation component. The software was implemented using MATLAB. As shown on the experiments in the previous section, it is difficult to judge an enhanced image result even with a subjective assessment. However, we claim that our method is more robust than the others in the sense that neither unrealistic colors nor over 
enhanced are produced. For future works, we plan to evaluate the methods using naturalness and colorfulness metrics on a database with hundreds of images collected from internet, such that a quantitative comparison can be performed.

However, there may be still some areas needs to be taken care of as the color enhancement needs to change or shift color using hue although these cases are exceptional and very rare.

\section{REFERENCES}

[1] C. Solomon, T. Breckon. 2011. Fundamentals of Digital Image Processing.

[2] Nishu, Sunil A. 2012, Quantifying the defect visibility in digital images by proper color space selection, International journal of engineering research and applications, vol.2, Issue 3, pp. 1764-1767.

[3] Raunaq M. and Utkarsh U., 2008, Hus-preserving color image enhancement without gamut problem, Term paper, pp. 1-6.

[4] Yusuf Abu S., Nijad Al-Najdawi, Sara T., 2011, Exploiting Hybrid methods for enhancing digital X-Ray Images, International Arab journal of information technology, vol. 8.

[5] Umesh R., Zhou W., Eero P. S., 2009, Quantifying color image distortions based on adaptive spatio-chromatic signal decompositions, IEEE international conference on image processing.
[6] Hana Al-Nuaim, Nouf A., 2011, A user perceived quality assessment of lossy compressed images, International journal of computer graphics, vol. 2, No. 2, pp. 23-36.

[7] R.C. Gonzalez and R.E. woods, 2007, Digital Image Processing, 3rd Edition, Prentice Hall, Upper Saddle River, NJ.

[8] Jian-feng Li, Kaun-Quan Wang, David Zhang, 2002, A New equation of saturation in RGB-TO-HIS conversion for more rapidity of computing, Proceedings of the international conference on machine learning and cybernertics, pp. 1493-1497.

[9] Papoulis, A., 1968, Systems and Transforms with Applications in Optics, New York: McGraw-Hill.

[10] Russ, J.C., 1995, the Image Processing Handbook. Second ed., Boca Raton, Florida: CRC Press.

[11] R. E. Blake, 1999, Partitioning Graph Matching with Constraints, Pattern Recognition, Vol 27, No.3, pp 439446.

[12] J. Foley, A. van Dam, S. Feiner and J. Hughes, 1990, Computer Graphics: Principles and Practice, Second Edition, Addison-Wesley, Reading, MA.

[13] R. E. Blake and P. Boros, 1995, The Extraction of Structural Features for Use in Computer Vision. Proceedings of the Second Asian Conference on Computer Vision, Singapore. 